\title{
SPECTRAL VISCOSITY APPROXIMATIONS TO MULTIDIMENSIONAL SCALAR CONSERVATION LAWS
}

\author{
GUI-QIANG CHEN, QIANG DU, AND EITAN TADMOR
}

\begin{abstract}
We study the spectral viscosity (SV) method in the context of multidimensional scalar conservation laws with periodic boundary conditions. We show that the spectral viscosity, which is sufficiently small to retain the formal spectral accuracy of the underlying Fourier approximation, is large enough to enforce the correct amount of entropy dissipation (which is otherwise missing in the standard Fourier method). Moreover, we prove that because of the presence of the spectral viscosity, the truncation error in this case becomes spectrally small, independent of whether the underlying solution is smooth or not. Consequently, the SV approximation remains uniformly bounded and converges to a measure-valued solution satisfying the entropy condition, that is, the unique entropy solution. We also show that the SV solution has a bounded total variation, provided that the total variation of the initial data is bounded, thus confirming its strong convergence to the entropy solution. We obtain an $L^{1}$ convergence rate of the usual optimal order one-half.
\end{abstract}

\section{THE SPECTRAL VISCOSITY APPROXIMATION}

We consider scalar conservation laws in several space dimensions $d, d \geq 1$,

$$
\partial_{t} u(x, t)+\partial_{x} \cdot f(u(x, t))=0, \quad f(u) \equiv\left(f^{1}(u), f^{2}(u), \ldots, f^{d}(u)\right),
$$

subject to initial data

$$
u(x, 0) \equiv u_{0}(x) \in L^{\infty}\left(T^{d}[0,2 \pi]\right),
$$

and augmented with the entropy condition (cf. $[12,17])$

$$
\partial_{t} U(u)+\partial_{x} \cdot F(u) \leq 0, \quad \forall U \text { convex }, \quad F(u) \equiv \int^{u} U^{\prime}(w) f^{\prime}(w) d w .
$$

The following abbreviations are used throughout the paper:

$$
\partial_{t}=\frac{\partial}{\partial t}, \quad \partial_{j}=\frac{\partial}{\partial x_{j}}, \quad \partial_{j k}^{2}=\frac{\partial^{2}}{\partial x_{j} \partial x_{k}}, \quad \partial_{x}^{s}=\left(\partial_{1}^{s}, \partial_{2}^{s}, \ldots, \partial_{d}^{s}\right) .
$$

We want to solve the $2 \pi$-periodic initial value problem, $(1.1 \mathrm{a})-(1.1 \mathrm{~b})$, by a spectral method. To this end, we approximate the spectral/pseudospectral

Received by the editor November 25, 1991 .

1991 Mathematics Subject Classification. Primary 35L65, 65M06, 65M12, 65M15.

Key words and phrases. Multidimensional conservation laws, spectral viscosity method, spectral accuracy, measure-valued solution, total variation, convergence, error estimate. 
projection of the exact entropy solution, $P_{N} u(\cdot, t)$, using an $N$-trigonometric polynomial, $u_{N}(x, t)=\sum_{|\xi| \leq N} \hat{u}_{\xi}(t) e^{i \xi \cdot x}$, which is governed by the semidiscrete approximation

$$
\partial_{t} u_{N}(x, t)+\partial_{x} \cdot P_{N} f\left(u_{N}(x, t)\right)=\varepsilon_{N} \sum_{j, k=1}^{d} \partial_{j k}^{2} Q_{N}^{j, k}(x, t) * u_{N}(x, t) .
$$

Together with one's favorite ODE solver, (1.2a) gives a fully discrete method for the approximate solutions of (1.1a). Discussion of the numerical advantages and actual implementation of this method will be made elsewhere. Our focus in this paper is on the convergence theory.

The left-hand side of (1.2a) is the standard Fourier approximation of (1.1a). Although this part of the approximation is spectrally accurate for the conservation law (1.1a), it lacks entropy dissipation, which is inconsistent with the entropy condition (1.1b). Consequently, the standard Fourier approximation of (1.1a) supports spurious Gibbs oscillations (once shock discontinuities are formed), which prevent strong convergence to the entropy solution of (1.1) (cf. $[19,20])$. To suppress these oscillations, without sacrificing the overall spectral accuracy, we augment the standard Fourier approximation on the right-hand side of (1.2a) by spectral viscosity, which consists of the following three ingredients:

- A vanishing viscosity amplitude, $\varepsilon_{N}$, of size

$$
\varepsilon_{N} \sim N^{-\theta}, \quad \theta<1 .
$$

- A viscosity-free spectrum of size $m_{N}>>1$,

$$
m_{N} \sim \frac{N^{\frac{\theta}{2}}}{(\log N)^{\frac{d}{2}}}, \quad \theta<1 .
$$

- A family of viscosity kernels, $Q_{N}^{j, k}(x, t)=\sum_{|\xi|=m_{N}}^{N} \widehat{Q}_{\xi}^{j, k}(t) e^{i \xi \cdot x}, \quad 1 \leq$ $j, k \leq d$, activated only on high wave numbers $|\xi| \geq m_{N}$, which can be conveniently implemented in the Fourier space as

$$
\begin{gathered}
\varepsilon_{N} \sum_{j, k=1}^{d} \partial_{j k}^{2} Q_{N}^{j, k} * u_{N}(x, t) \equiv-\varepsilon_{N} \sum_{|\xi|=m_{N}}^{N}\left\langle\widehat{Q}_{\xi} \xi, \xi\right\rangle \hat{u}_{\xi}(t) e^{i \xi \cdot x}, \\
\left\langle\hat{Q}_{\xi} \xi, \xi\right\rangle \equiv \sum_{j, k=1}^{d} \hat{Q}_{\xi}^{j, k}(t) \xi_{j} \xi_{k} .
\end{gathered}
$$

The viscosity kernels we deal with, $Q_{N}^{j, k}(x, t)$, are assumed to be spherically symmetric, that is, $\widehat{Q}_{\xi}^{j, k}=\widehat{Q}_{p}^{j, k}, \forall|\xi|=p$, with monotonically increasing Fourier coefficients, $\hat{Q}_{p}^{j, k}$, that satisfy

$$
\left|\hat{Q}_{p}^{j, k}-\delta_{j k}\right| \leq \text { Const } \frac{m_{N}^{2}}{p^{2}}, \quad \forall p \geq m_{N} .
$$

The main purpose of the spectral viscosity is to achieve a compromise between two conflicting requirements. We recall (cf. [7]) that the use of the 
spectral/pseudo-spectral projections yields a spectrally small error in the sense that

$$
\left\|\left(I-P_{N}\right) f\left(u_{N}\right)\right\| \leq \text { Const } N^{-s}\left\|\partial_{x}^{s} u_{N}\right\|, \quad \forall s \geq 0 .
$$

The additional spectral viscosity is also spectrally small, since

$$
\varepsilon_{N}\left\|\sum_{j, k=1}^{d} \partial_{j k}^{2} Q_{N}^{j, k} * u_{N}(\cdot, t)\right\| \leq \text { Const } N^{-\frac{s \theta}{2}}\left\|\partial_{x}^{s} u_{N}(\cdot, t)\right\|, \quad \forall s \geq 2 .
$$

Thus, on the one hand the spectral viscosity is small enough to retain the formal spectral accuracy of the overall approximation, while on the other hand the spectral viscosity is sufficiently large to enforce the correct amount of entropy dissipation that is missing in the standard Fourier method, that is, $\varepsilon_{N}=0$ (see $\S 2)$. In fact, the smallest scale of the SV approximation (1.2a) is order $\varepsilon_{N}$. It follows that, because of the presence of the SV in (1.2a), the spectral decay of the truncation error on the left of (1.3) is independent of the smoothness of the underlying solution. In $\S 3$ we show that the SV, although spectrally small, is only an $L^{p}$-bounded perturbation of the standard vanishing viscosity. This fact enables us to show in $\S 4$ that the SV solution remains uniformly bounded and that its weak limit is a measure-valued solution consistent with the entropy condition corresponding to (1.1b). Hence, DiPerna's uniqueness theorem [5] combined with the finiteness of propagation speed implies that $u_{N}$ converges to the unique entropy solution of $(1.1 \mathrm{a})-(1.1 \mathrm{~b})$. For the reduction theorems for the measure-valued solutions to hyperbolic systems of conservation laws, we also refer the reader to $[2,3,6,16]$. An alternative, independent convergence proof of the SV method is derived in $\S 5$ from its total-variation boundedness, provided the total variation of the initial data is bounded. We conclude in $\S 6$ with an $L^{1}$-convergence rate estimate of the usual optimal order one-half.

\section{SPECTRALly SMALl TRUNCATION ERROR}

The SV method (1.2a) approximates the exact spatial fluxes in (1.1a) by their (pseudo)spectral projections. This approach leads to the truncation error $\partial_{x} \cdot\left(I-P_{N}\right) f\left(u_{N}\right)$. In this section we show that, because of the presence of the spectral viscosity, the truncation error is spectrally small independent of whether the underlying solution is smooth or not.

Our discussion proceeds in three steps; detailed proofs are left to $\S 7$.

Step 1. We begin with the following two facts.

- A straightforward a priori bound states that for all $s \geq r \geq 0$ there holds:

$$
\left\|\partial_{x}^{r} \cdot\left(I-P_{N}\right) f\left(u_{N}\right)\right\|=\left(\sum_{|\xi|>N}|\xi|^{2 r}\left|\widehat{f\left(u_{N}\right)}\right|^{2}\right)^{\frac{1}{2}} \leq \frac{1}{N^{s-r}}\left\|\partial_{x}^{s} \cdot f\left(u_{N}\right)\right\| .
$$

- Consider the right-hand side of (2.1). Here we claim (and prove in $\S 7$ ) that the derivatives of a sufficiently smooth flux, $f(u)$, are bounded above by the derivatives of $u$. That is, there exist constants $\mathscr{K}_{S}$ (depending on $\left|u_{N}\right|_{L^{\infty}} \equiv$ 
$\left\|u_{N}\right\|_{L_{\text {loc }}^{\infty}(x, t)}$ and $|f|_{C^{k}} \equiv\left\|\partial_{u}^{k} f(u)\right\|_{L^{\infty}\left(\Omega_{N}\right)}$, where $\left.\Omega_{N}=\left\{u:|u| \leq\left|u_{N}\right|_{\infty}\right\}\right)$, such that the following estimate holds:

$$
\begin{gathered}
\left\|\partial_{x}^{s} \cdot f\left(u_{N}\right)\right\| \leq \mathscr{K}_{s}\left\|\partial_{x}^{s} u_{N}\right\|, \\
\mathscr{K}_{s} \sim \sum_{k=1}^{s}|f|_{C^{k}} \cdot\left|u_{N}\right|_{L^{\infty}}^{k-1}, \quad s=1,2, \ldots
\end{gathered}
$$

Using (2.1) followed by (2.2), we conclude the first step with the estimate

$$
\begin{aligned}
& \mid \partial_{x}^{r} \cdot\left(I-P_{N}\right) f\left(u_{N}\right)\left\|\leq \frac{\mathscr{K}_{s}}{N^{s-r}}\right\| \partial_{x}^{s} u_{N} \|, \\
& \mathscr{K}_{s} \sim \sum_{k=1}^{s}|f|_{C^{k}} \cdot\left|u_{N}\right|_{L^{\infty}}^{k-1}, \quad s \geq r \geq 0 .
\end{aligned}
$$

Step 2. The inequality (2.3) is the usual spectral accuracy estimate associated with the (pseudo)spectral projections (see (1.3)). The inequality states that the truncation error (and its derivatives) decays as rapidly as the smoothness of $u_{N}$ permits. Of course, the derivatives of an arbitrary $N$-trigonometric polynomial, $\partial_{x}^{s} u_{N}$, may grow as fast as $N^{s}$, in which case nothing is gained from (2.3). In the present context, however, the spatial derivatives of the SV approximation $\partial_{x}^{s} u_{N}$ grow at the slower rate of $\varepsilon_{N}^{-s}$. This is the content of the main a priori estimate of this section, whose proof is left for $\S 7$. We summarize the estimate in the following theorem.

Theorem 2.1. Consider the $S V$ approximation (1.2a) and (1.2d) with SV parameters $\left(\varepsilon_{N}, m_{N}\right)$, which satisfy, in agreement with $(1.2 \mathrm{~b})-(1.2 \mathrm{c})$,

$$
\varepsilon_{N}>\frac{2 \mathscr{R}_{s+1}}{N}, \text { and } \varepsilon_{N} \cdot m_{N}^{2}(\log N)^{d} \leq \text { Const . }
$$

Then there exists a constant $\mathscr{B}_{s}\left(\sim \prod_{k=1}^{s} \mathscr{K}_{k}\right.$ for $\left.s \geq 1\right)$ such that the following estimate holds:

$$
\begin{gathered}
\varepsilon_{N}^{s}\left\|\partial_{x}^{s} u_{N}(\cdot, t)\right\|_{L^{2}(x)}+\varepsilon_{N}^{s+\frac{1}{2}}\left\|\partial_{x}^{s+1} u_{N}\right\|_{L^{2}(x,[0, t])} \\
\leq \mathscr{B}_{s}+\text { Const } \varepsilon_{N}^{s}\left\|\partial_{x}^{s} u_{N}(\cdot, 0)\right\|_{L^{2}(x)} .
\end{gathered}
$$

Remark. Theorem 2.1 with $s=0$ is the usual a priori estimate on the entropy production rate

$$
\left\|u_{N}(\cdot, t)\right\|_{L^{2}(x)}+\sqrt{\varepsilon_{N}}\left\|\partial_{x}^{s} u_{N}\right\|_{L^{2}(x,[0, t])} \leq \text { Const }\left\|u_{N}(\cdot, 0)\right\|_{L^{2}(x)} \cdot
$$

Step 3. Assume that our data (the flux, $f(u)$, and the initial conditions, $\left.u_{N}(\cdot, 0)\right)$ satisfy the following conditions:

- The flux $f(u)$ is sufficiently smooth; that is, $|f|_{C^{s}}<\infty$ for sufficiently large $s$ :

- The smallest scale of the initial condition is of order $\varepsilon_{N}$; that is,

$$
\varepsilon_{N}^{s}\left\|\partial_{x}^{s} u_{N}(\cdot, 0)\right\|_{L^{2}(x)} \leq \text { Const. }
$$

Then, Theorem 2.1 shows that the smallest scale of the SV approximation $u_{N}(\cdot, t)$ remains of order $\varepsilon_{N}$. Together with $(2.3)$, this implies that for $\frac{1}{N \varepsilon_{N}} \sim$ $N^{-(1-\theta)}=o(1)$, the truncation error of the SV approximation is spectrally 
small, independent of whether the underlying solution is smooth or not. This extends a similar one-dimensional result of $[15,13]$. For later reference, we state our final corollary.

Corollary 2.2. Let $u_{N}$ denote the $S V$ solution of $(1.2 \mathrm{a})-(1.2 \mathrm{~d})$, with initial conditions satisfying (2.7). Then the following spectral decay estimates of the truncation error hold:

$$
\begin{gathered}
\left\|\partial_{x}^{r} \cdot\left(I-P_{N}\right) f\left(u_{N}(\cdot, t)\right)\right\|_{L^{2}(x)} \leq \text { Const } \mathscr{B}_{S} N^{-s_{r}}, \quad s_{r} \equiv(1-\theta) s-r \\
\left\|\partial_{x}^{r} \cdot\left(I-P_{N}\right) f\left(u_{N}\right)\right\|_{L^{2}(x,[0, t])} \leq \text { Const } \mathscr{B}_{s} N^{-\left(s_{r}+\frac{\theta}{2}\right)}, \quad \forall s \geq 1 .
\end{gathered}
$$

Remarks. 1. Using the Sobolev inequality, one can convert this $L^{2}$-type estimate (2.8) into a spectral decay estimate in the uniform norm, for example,

$$
\left\|\partial_{x}^{r} \cdot\left(I-P_{N}\right) f\left(u_{N}\right)\right\|_{L^{\infty}(x,[0, t])} \leq \text { Const } \mathscr{B}_{s} N^{-s_{r}+\frac{d}{2}} .
$$

2. Observe that the polynomial decay rate in (2.9), $s_{r}=(1-\theta) s-r$, can be made as large as the $C^{s}$-smoothness of $f(u)$ permits. For example, we have

$$
\begin{aligned}
\left\|\partial_{x} \cdot\left(I-P_{N}\right) f\left(u_{N}\right)\right\|_{L^{\infty}(x,[0, t]) \leq} & \operatorname{Const} \frac{\mathscr{B} s}{N}, \\
& \forall f \in C^{s}, \quad s \geq \frac{2+d}{2(1-\theta)} .
\end{aligned}
$$

The smoothness requirement on the right of $(2.10)$ will be sufficient for the estimates derived throughout the rest of the paper.

3. The critical Sobolev exponents $s_{r}$ in (2.9) are not optimal; careful $L^{p}$ iterations enable one to obtain (2.9) with $s_{0}=(1-\theta) s+1-\theta-\frac{d}{2} \geq 0$. Thus, for example, in the one-dimensional case, one may use the basic entropy production bound (2.6) (corresponding to $s=0$ ), in order to conclude that the truncation error tends uniformly to zero with viscosity amplitude $\varepsilon_{N} \sim$ $N^{-\theta}, \theta<\frac{1}{2}$ (cf. $[13,21]$ ).

\section{SPECTRAL VISCOSITY VERSUS VANISHING VISCOSITY}

In this section we show that the spectral viscosity $\varepsilon_{N} \sum_{j, k=1}^{d} \partial_{j k}^{2} Q_{N}^{j, k} * u_{N}-$ though spectrally small according to (1.4) -is only an $L^{p}$-bounded perturbation of the standard vanishing viscosity, $\varepsilon_{N} \Delta u_{N}$.

We begin by taking a closer look at the SV operator on the right of (1.2a). We set

$$
\widehat{R}_{\xi}^{j, k} \equiv \widehat{R}_{\xi}^{j, k}(t)=\left\{\begin{array}{l}
\delta_{j k}, \quad|\xi| \leq m_{N}, \\
\delta_{j k}-\widehat{Q}_{\xi}^{j, k}(t), \quad|\xi|>m_{N},
\end{array}\right.
$$

and note that the corresponding smoothing kernel, $R_{N}^{j, k} \equiv \sum_{|\xi| \leq N} \widehat{R}_{\xi}^{j, k} e^{i \xi \cdot x}$, complements the SV kernel, $Q_{N}^{j, k}=\sum_{|\xi|=m_{N}}^{N} \widehat{Q}_{\xi}^{j, k} e^{i \xi \cdot x}$, to the full Laplacian, 
that is,

$$
\sum_{j, k=1}^{d} \partial_{j k}^{2} Q_{N}^{j, k} *+\sum_{j, k=1}^{d} \partial_{j k}^{2} R_{N}^{j, k} * \equiv \Delta
$$

The following lemma provides us with an upper bound on the $L^{1}$-size of the smoothing kernel, $\sum_{j, k=1}^{d} \partial_{j}^{r} \partial_{k}^{s-r} R_{N}^{j, k}(\cdot, t)$.

Lemma 3.1. Consider the real, spherically symmetric SV kernels,

$$
Q_{N}^{j, k}(x, t)=\sum_{p \geq m_{N}}^{N} \widehat{Q}_{p}^{j, k} \sum_{|\xi|=p} e^{i \xi \cdot x}
$$

with monotonically increasing Fourier coefficients, $\widehat{Q}_{p}^{j, k}$, satisfying (1.2d). Then the following estimate holds:

$$
\left\|\sum_{j, k=1}^{d} \partial_{j}^{r} \partial_{k}^{s-r} R_{N}^{j, k}(\cdot, t)\right\|_{L^{1}(x)} \leq \text { Const } m_{N}^{s}(\log N)^{d}, \quad 0 \leq r \leq s \leq 2 .
$$

Remarks. 1. Lemma 3.1, which is the multidimensional generalization of Lemma A.1 in [13], shows that derivatives of the smoothing kernel, $\sum_{j, k=1}^{d} R_{N}^{j, k}(\cdot, t)$, grow like those of a trigonometric polynomial of degree $m_{N}$.

2. The extra logarithmic factor on the right of (3.3) can be avoided if the SV Fourier coefficients, $\widehat{Q}_{p}^{j, k}$, satisfy the concavity condition, $\widehat{Q}_{p+1}^{j, k}-2 \widehat{Q}_{p}^{j, k}+$ $\widehat{Q}_{p-1}^{j, k} \leq 0$.

Proof of Lemma 3.1. Let $D_{p}\left(x_{i}\right)=\frac{\sin \left(p+\frac{1}{2}\right) x_{i}}{\sin \frac{1}{2} x_{i}}$. Then we have

$$
\sum_{|\xi|=p} e^{i \xi \cdot x} \equiv \mathbb{D}_{p}(x)-\mathbb{D}_{p-1}(x),
$$

where $\mathbb{D}_{p}(x)=\sum_{|\xi| \leq p} e^{i \xi \cdot x}$ is the multidimensional Dirichlet kernel, $\mathbb{D}_{p}(x)=$ $\prod_{i=1}^{d} D_{p}\left(x_{i}\right)$. This fact enables us to write (with $\mathbb{D}_{-1}(x) \equiv 0$ )

$$
R_{N}^{j, k}(x, t)=\sum_{p=0}^{N} \widehat{R}_{p}^{j, k}(t) \sum_{|\xi|=p} e^{i \xi \cdot x}=\sum_{p=0}^{N} \widehat{R}_{p}^{j, k}(t)\left[\mathbb{D}_{p}(x)-\mathbb{D}_{p-1}(x)\right] .
$$

Setting $\widehat{R}_{N+1}^{j, k}=0$, we use summation by parts of the right-hand side (recall that $\widehat{R}_{p}^{j, k}=\delta_{j k}, p \leq m_{N}$ ) to obtain

$$
\begin{aligned}
\partial_{j}^{r} \partial_{k}^{s-r} R_{N}^{j, k}(x, t) & =\sum_{p=0}^{N} \widehat{R}_{p}^{j, k} \partial_{j}^{r} \partial_{k}^{s-r}\left[\mathbb{D}_{p}(x)-\mathbb{D}_{p-1}(x)\right] \\
& =\sum_{p=m_{N}}^{N}\left(\widehat{R}_{p}^{j, k}-\widehat{R}_{p+1}^{j, k}\right) \partial_{j}^{r} \partial_{k}^{s-r} \mathbb{D}_{p}(x) .
\end{aligned}
$$


Next, since

$$
\left\|\frac{d^{s}}{d x_{i}^{s}} D_{p}\left(x_{i}\right)\right\|_{L^{1}}= \begin{cases}\mathscr{O}(\log p), & s=0, \\ \mathscr{O}\left(p^{s}\right), & s=1,2, \ldots,\end{cases}
$$

we have $\left\|\partial_{j}^{r} \partial_{k}^{s-r} \mathbb{D}_{p}(x)\right\|_{L^{1}} \leq$ Const $p^{s}(\log p)^{d_{s}}, \quad d_{s}=d-1+(1-s)^{+}$. Using this and the fact that $\widehat{R}_{p}^{j, k}$ are monotonically decreasing, we conclude from (3.4)

$$
\begin{aligned}
& \left\|\partial_{j}^{r} \partial_{k}^{s-r} R_{N}^{j, k}(\cdot, t)\right\|_{L^{1}} \\
& \quad \leq \text { Const }\left[\sum_{p=m_{N}}^{N-1}\left(\widehat{R}_{p}^{j, k}-\widehat{R}_{p+1}^{j, k}\right) p^{s}(\log p)^{d_{s}}+\left|\widehat{R}_{N}^{j, k}\right| \cdot N^{s}(\log N)^{d_{s}}\right] .
\end{aligned}
$$

One more summation by parts on the right of the last inequality yields

$$
\begin{aligned}
& \left\|\partial_{j}^{r} \partial_{k}^{s-r} R_{N}^{j, k}(\cdot, t)\right\|_{L^{1}} \\
& \quad \leq \text { Const }\left[\sum_{p=m_{N}}^{N} \widehat{R}_{p}^{j, k}\left[p^{s}(\log p)^{d_{s}}-(p-1)^{s}(\log (p-1))^{d_{s}}\right]+m_{N}^{2}(\log N)^{d_{s}}\right] .
\end{aligned}
$$

Finally, since by $(1.2 \mathrm{~d}), \widehat{R}_{p}^{j, k} \equiv \delta_{j k}-\widehat{Q}_{p}^{j, k} \leq m_{N}^{2} / p^{2}$, we conclude

$$
\begin{aligned}
\left\|\partial_{j}^{r} \partial_{k}^{s-r} R_{N}^{j, k}(\cdot, t)\right\|_{L^{1}} & \leq \text { Const } m_{N}^{2}(\log N)^{d_{s}} \cdot \sum_{p=m_{N}}^{N} \frac{2}{p^{3-s}} \\
& \leq \text { Const } m_{N}^{s}(\log N)^{d}, \quad \forall s \leq 2 .
\end{aligned}
$$

Young's inequality followed by Lemma 3.1 with $(r, s)=(1,2)$ implies our final corollary, which confirms the statement at the beginning of this section.

Corollary 3.2. Consider the real, spherically symmetric SV kernels,

$$
Q_{N}^{j, k}(x, t)=\sum_{p \geq m_{N}}^{N} \widehat{Q}_{p}^{j, k} \sum_{|\xi|=p} e^{i \xi \cdot x}
$$

with monotonically increasing Fourier coefficients, $\widehat{Q}_{p}^{j, k}$, satisfying (1.2d). Denote

$$
c_{N} \sim \varepsilon_{N} m_{N}^{2}(\log N)^{d} \leq \text { Const } .
$$

Then the following estimate holds:

$$
\varepsilon_{N}\left\|\sum_{j, k=1}^{d} \partial_{j k}^{2} R_{N}^{j, k} * u_{N}(\cdot, t)\right\|_{L^{p}} \leq c_{N}\left\|u_{N}(\cdot, t)\right\|_{L^{p}}, \quad \forall p \geq 1 .
$$

\section{ConVergence OF THE SV METHOD}

In the first part of this section we prove that the SV approximation, (1.2a)(1.2d), is uniformly bounded. 
Lemma 4.1 ( $L^{\infty}$-stability). There exists a constant such that

$$
\left\|u_{N}(\cdot, t)\right\|_{L^{\infty}(x)} \leq \text { Const }\left\|u_{N}(\cdot, 0)\right\|_{L^{\infty}(x)}, \quad \forall t \leq T .
$$

In the second part of this section we show that the SV solution is consistent with the entropy condition (1.1b) for all convex entropy pairs $(U, F)$.

Lemma 4.2 (Entropy consistency). There exists a vanishing sequence, $e_{N}$, such that

$$
\partial_{t} U\left(u_{N}\right)+\partial_{x} \cdot F\left(u_{N}\right) \leq e_{N} \rightarrow 0 \text { in } \mathscr{D}^{\prime} .
$$

The detailed proof of Lemmas 4.1 and 4.2 is postponed to the end of this section. Granted the $L^{\infty}$-stability and the entropy consistency, we can combine DiPerna's uniqueness result for measure-valued solutions [5] with the finiteness of propagation speed (see also [18] for the case of bounded domains) to conclude the following theorem.

Theorem 4.3. Let $u_{N}$ be the solution of the $S V$ approximation (1.2a)-(1.2d), subject to bounded initial conditions satisfying

$$
\left\|u_{N}(\cdot, 0)\right\|_{L^{\infty}(x)}+\varepsilon_{N}^{s}\left\|\partial_{x}^{s} u_{N}(\cdot, 0)\right\|_{L^{2}(x)} \leq \text { Const } .
$$

Then $u_{N}$ converges strongly to the unique entropy solution of (1.1a)-(1.1b).

We now turn to the promised proofs of the $L^{\infty}$-stability and the entropy consistency.

Proof of Lemma 4.1. Using (3.2), we can rewrite the SV approximation (1.2a) in the form

$$
\partial_{t} u_{N}+\partial_{x} \cdot P_{N} f\left(u_{N}\right)-\varepsilon_{N} \Delta u_{N}=\varepsilon_{N} \sum_{j, k=1}^{d} \partial_{j k}^{2} R_{N}^{j, k} * u_{N}
$$

or, equivalently,

$$
\partial_{t} u_{N}+\partial_{x} \cdot f\left(u_{N}\right)-\varepsilon_{N} \Delta u_{N}=\varepsilon_{N} \sum_{j, k=1}^{d} \partial_{j k}^{2} R_{N}^{j, k} * u_{N}+\partial_{x} \cdot\left(I-P_{N}\right) f\left(u_{N}\right)
$$

Integrating against $p u_{N}^{p-1}$ and letting $p \uparrow \infty$, we are led to the maximum principle associated with the parabolic left-hand side of $(4.3 \mathrm{~b})$, which reads

$$
\begin{aligned}
\frac{d}{d t}\left\|u_{N}(\cdot, t)\right\|_{L^{\infty}(x)} \leq & \left\|\partial_{x} \cdot\left(I-P_{N}\right) f\left(u_{N}(\cdot, t)\right)\right\|_{L^{\infty}(x)} \\
& +\varepsilon_{N}\left\|\sum_{j, k=1}^{d} \partial_{j k}^{2} R_{N}^{j, k} * u_{N}(\cdot, t)\right\|_{L^{\infty}(x)} .
\end{aligned}
$$

It remains to bound from above the two expressions on the right of (4.4). 
We now fix $s=\frac{2+d}{2(1-\theta)}$. Corollary 2.2 implies, consult (2.9), that the first term on the right of (4.4) does not exceed

$$
\left\|\partial_{x} \cdot\left(I-P_{N}\right) f\left(u_{N}(\cdot, t)\right)\right\|_{L^{\infty}(x)} \leq \frac{\mathscr{B}_{s}}{N}, \quad \mathscr{B}_{s} \sim \prod_{k=1}^{s} \mathscr{K}_{k} \leq \text { Const }\left\|u_{N}\right\|_{L^{\infty}(x,[0, t])}^{\frac{s^{2}}{2}} .
$$

By equation (3.6) with $p=\infty$, the second term on the right of (4.4) does not exceed

$$
\begin{array}{r}
\varepsilon_{N}\left\|\sum_{j, k=1}^{d} \partial_{j k}^{2} R_{N}^{j, k} * u_{N}(\cdot, t)\right\|_{L^{\infty}(x)} \leq c_{N}\left\|u_{N}\right\|_{L^{\infty}(x,[0, t])}, \\
c_{N} \sim \varepsilon_{N} m_{N}^{2}(\log N)^{d} \leq \text { Const } .
\end{array}
$$

Equipped with the last two upper bounds, we return to the inequality (4.4), which tells us that the growth of $\left|u_{N}(t)\right|_{\infty} \equiv\left\|u_{N}\right\|_{L^{\infty}(x,[0, t])}$ is governed by

$$
\frac{d}{d t}\left|u_{N}(t)\right|_{\infty} \leq c_{N}\left|u_{N}(t)\right|_{\infty}+\frac{\text { Const }}{N}\left|u_{N}(t)\right|_{\infty}^{\frac{s^{2}}{2}}
$$

which in turn implies that

$$
\left|u_{N}(t)\right|_{\infty} \leq e^{c_{N} t}\left|u_{N}(0)\right|_{\infty} \cdot\left(1-\frac{\left(e^{c_{N} t}\left|u_{N}(0)\right|_{\infty}\right)^{\frac{s^{2}}{2}}}{N c_{N}}\right)^{-\frac{2}{s^{2}}}
$$

We conclude that for $t \leq \mathscr{O}(\ln N)$, the SV solution remains bounded by Const $e^{c_{N} t}\left|u_{N}(0)\right|_{\infty}$.

We close this section with the following proof.

Proof of Lemma 4.2. Multiplying (4.3b) by $U^{\prime}\left(u_{N}\right)$, we obtain

$$
\begin{aligned}
\partial_{t} U & \left(u_{N}\right)+\partial_{x} \cdot F\left(u_{N}\right)-\varepsilon_{N} \Delta U\left(u_{N}\right) \\
\leq & \varepsilon_{N} U^{\prime}\left(u_{N}\right) \sum_{j, k=1}^{d} \partial_{j k}^{2} R_{N}^{j, k} * u_{N}+U^{\prime}\left(u_{N}\right) \partial_{x} \cdot\left(I-P_{N}\right) f\left(u_{N}\right) \\
= & \sum_{j, k=1}^{d} \partial_{j}\left(\varepsilon_{N} U^{\prime}\left(u_{N}\right) R_{N}^{j, k} * \partial_{k} u_{N}\right)-\varepsilon_{N} U^{\prime \prime}\left(u_{N}\right) \sum_{j, k=1}^{d} \partial_{j} u_{N} R_{N}^{j, k} * \partial_{k} u_{N} \\
& +\partial_{x} \cdot\left(U^{\prime}\left(u_{N}\right)\left(I-P_{N}\right) f\left(u_{N}\right)\right)-U^{\prime \prime}\left(u_{N}\right) \partial_{x} u_{N} \cdot\left(I-P_{N}\right) f\left(u_{N}\right) \\
\equiv & \sum_{j, k=1}^{d} \partial_{j}\left(I_{j k}\left(u_{N}\right)\right)-\operatorname{II}\left(u_{N}\right)+\partial_{x} \cdot \operatorname{III}\left(u_{N}\right)-\operatorname{IV}\left(u_{N}\right)
\end{aligned}
$$

We claim that the four terms on the right of (4.5) tend to zero. Below, we abbreviate $\left\|U^{\prime}\right\|_{L^{\infty}}=\left\|U^{\prime}(u)\right\|_{L^{\infty}\left(\Omega_{N}\right)}$, etc.

First, from (2.6) we have

$$
\begin{aligned}
\left\|\mathbf{I}_{j k}\left(u_{N}\right)\right\|_{L_{\text {loc }}^{2}(x, t)} & \leq \varepsilon_{N}\left\|U^{\prime}\right\|_{L^{\infty}} \max _{|\xi| \leq N}\left|\hat{R}_{\xi}^{j, k}\right| \cdot\left\|\partial_{k} u_{N}\right\|_{L_{\text {loc }}^{2}(x, t)} \\
& \leq \varepsilon_{N}\left\|U^{\prime}\right\|_{L^{\infty}}\left\|\partial_{x} u_{N}\right\|_{L_{\text {loc }}^{2}(x, t)} \leq \text { Const } \sqrt{\varepsilon_{N}} \rightarrow 0
\end{aligned}
$$


Next, since $\left|\left\langle\widehat{R}_{\xi} \xi, \xi\right\rangle\right| \leq$ Const $|\xi|^{2}$, we find

$$
\begin{aligned}
\left\|\mathrm{II}\left(u_{N}\right)\right\|_{L_{\text {loc }}^{2}(x, t)} & \leq \varepsilon_{N}\left\|U^{\prime \prime}\right\|_{L^{\infty}}\left(\int_{t=0}^{T} \sum_{|\xi| \leq N}\left|\left\langle\widehat{R}_{\xi}(t) \xi, \xi\right\rangle\right| \cdot\left|\hat{u}_{\xi}(t)\right|^{2} d t\right)^{\frac{1}{2}} \\
& \leq \text { Const } \varepsilon_{N}\left\|U^{\prime \prime}\right\|_{L^{\infty}}\left\|\partial_{x} u_{N}\right\|_{L_{\text {loc }}^{2}(x, t)} \leq \text { Const } \sqrt{\varepsilon_{N}} \rightarrow 0 .
\end{aligned}
$$

The inequality $(2.8 \mathrm{~b})$ with $(r, s)=(0,1)$ implies

$$
\begin{aligned}
\left\|\operatorname{III}\left(u_{N}\right)\right\|_{L_{\text {loc }}^{2}(x, t)} & \leq\left\|U^{\prime}\right\|_{L^{\infty}} \cdot\left\|\left(I-P_{N}\right) f\left(u_{N}\right)\right\|_{L_{\text {loc }}^{2}(x, t)} \\
& \leq\left\|U^{\prime}\right\|_{L^{\infty}} \cdot \frac{\mathscr{K}_{1}}{N}\left\|\partial_{x} u_{N}\right\|_{L_{\text {loc }}^{2}(x, t)} \leq \text { Const } N^{-\left(1-\frac{\theta}{2}\right)} \rightarrow 0 .
\end{aligned}
$$

Repeating the previous arguments, we conclude the proof of (4.2) with

$$
\begin{aligned}
\left\|\operatorname{IV}\left(u_{N}\right)\right\|_{L_{\text {loc }}^{1}(x, t)} & \leq\left\|U^{\prime \prime}\right\|_{L^{\infty}} \cdot\left\|\partial_{x} u_{N}\right\|_{L^{2}(x,[0, t])} \cdot\left\|\left(I-P_{N}\right) f\left(u_{N}\right)\right\|_{L^{2}(x,[0, t])} \\
& \leq \frac{\mathscr{R}_{1}}{N}\left\|\partial_{x} u_{N}\right\|_{L_{\text {loc }}^{2}(x, t)}^{2} \leq \text { Const } N^{-(1-\theta)} \rightarrow 0 .
\end{aligned}
$$

\section{TOTAL-VARIATION BOUNDEDNESS}

Consider the SV approximation (4.3b)

(5.1) $\partial_{t} u_{N}+\partial_{x} \cdot f\left(u_{N}\right)-\varepsilon_{N} \Delta u_{N}=\partial_{x} \cdot\left(I-P_{N}\right) f\left(u_{N}\right)+\varepsilon_{N} \sum_{j, k=1}^{d} R_{N}^{j, k} * \partial_{j k}^{2} u_{N}$

On the left we have the usual $L^{1}$-stable viscosity approximation of (1.1a)(1.1b). The first and second terms on the right represent, respectively, the spectrally small truncation error and, by Corollary 3.2 , the $L^{p}$-bounded perturbation of the SV operator. It follows that the SV approximation shares the $L^{1}$-stability and, consequently, the total-variation boundedness of the usual viscosity approximation. Details of the one-dimensional case can be found in [22]. To demonstrate the above claims in the multidimensional case, we now turn to a direct proof of the latter total-variation bound.

Spatial differentiation of $(5.1)$ yields

$$
\begin{aligned}
\partial_{t} \partial_{i} u_{N}+\partial_{x} \cdot\left(f^{\prime}\left(u_{N}\right) \partial_{i} u_{N}\right)-\varepsilon_{N} \Delta \partial_{i} u_{N} \\
=\partial_{i} \partial_{x} \cdot\left(I-P_{N}\right) f\left(u_{N}\right)+\varepsilon_{N} \sum_{j, k=1}^{d} \partial_{j k}^{2} R_{N}^{j, k} * \partial_{i} u_{N}
\end{aligned}
$$

Integrating this against $\operatorname{sign} \partial_{i} u_{N}$ and using (3.6) with $p=1$, we obtain

$$
\frac{d}{d t}\left\|\partial_{i} u_{N}(\cdot, t)\right\|_{L^{1}(x)} \leq \text { Const }\left\|\partial_{x}^{2} \cdot\left(I-P_{N}\right) f\left(u_{N}\right)\right\|_{L^{1}(x)}+c_{N}\left\|\partial_{i} u_{N}(\cdot, t)\right\|_{L^{1}(x)} .
$$

Integration of the last inequality implies that the total variation of the SV solu- 
tion, $\left\|u_{N}(\cdot, t)\right\|_{B V(x)} \equiv \sum_{i=1}^{d}\left\|\partial_{i} u_{N}\right\|_{L^{1}(x)}$, does not exceed

$$
\left\|u_{N}(\cdot, t)\right\|_{B V(x)} \leq e^{c_{N} t}\left[\left\|u_{N}(\cdot, 0)\right\|_{B V(x)}+\left\|\partial_{x}^{2} \cdot\left(I-P_{N}\right) f\left(u_{N}\right)\right\|_{L^{1}(x,[0, t])}\right] .
$$

By taking into account the spectral decay of the truncation error $(2.8 \mathrm{a})$, we conclude the following theorem.

Theorem 5.1. Let $u_{N}$ be the solution of the $S V$ approximation (1.2a)-(1.2d), subject to initial conditions (2.7). Then $u_{N}$ has a bounded variation, and the following estimate holds:

$$
\begin{array}{r}
\left\|u_{N}(\cdot, t)\right\|_{B V(x)} \leq e^{c_{N} t}\left[\left\|u_{N}(\cdot, 0)\right\|_{B V(x)}+\mathscr{O}\left(\sqrt{t} N^{-s_{2}}\right)\right], \\
s_{2}=(1-\theta) s-2>0 .
\end{array}
$$

\section{CONVERgenCE REVISITED WITH ERroR ESTIMATE}

In this section we revisit the question of convergence of the $\mathrm{SV}$ approximation to the unique entropy solution of (1.1a)-(1.1b). An affirmative answer to this question was already given in $\S 4$, where we used the $L^{\infty}$-bound together with the entropy consistency of the SV approximation. Alternatively, one may use the $L^{\infty}$-bound together with the total-variation boundedness to conclude the convergence of the SV approximation. Moreover, in this section we show how one can use the total-variation boundedness to obtain an $L^{1}$-convergence rate estimate of the usual optimal order one-half. We proceed along the lines of the one-dimensional argument in [15].

It is well known that the solution of the usual viscosity approximation

$$
\partial_{t} v^{\varepsilon_{N}}+\partial_{x} \cdot f\left(v^{\varepsilon_{N}}\right)=\varepsilon_{N} \Delta v^{\varepsilon_{N}},
$$

satisfies an $L^{1}$-convergence rate estimate of order one-half (cf. [11, 4, 14]):

$$
\left\|v^{\varepsilon_{N}}(\cdot, t)-u(\cdot, t)\right\|_{L^{1}(x)} \leq \text { Const } \sqrt{\varepsilon_{N}}, \quad 0 \leq t \leq T .
$$

We claim that the $L^{1}$-error between the SV approximation, $u_{N}$, and the viscosity approximation, $v^{\varepsilon_{N}}$, is of the same order $\mathscr{O}\left(\sqrt{\varepsilon_{N}}\right)$. Indeed, subtracting (6.1) from the SV approximation (4.3b), we find

$$
\begin{gathered}
\partial_{t}\left(u_{N}-v^{\varepsilon_{N}}\right)+\partial_{x} \cdot\left(f\left(u_{N}\right)-f\left(v^{\varepsilon_{N}}\right)\right)-\varepsilon_{N} \Delta\left(u_{N}-v^{\varepsilon_{N}}\right) \\
=\varepsilon_{N} \sum_{j, k=1}^{d} \partial_{j} R_{N}^{j, k} * \partial_{k} u_{N}+\partial_{x} \cdot\left(I-P_{N}\right) f\left(u_{N}\right) .
\end{gathered}
$$

Integrated against $\operatorname{sign}\left(u_{N}-v^{\varepsilon_{N}}\right)$, the last equality gives us

$$
\begin{aligned}
\frac{d}{d t}\left\|u_{N}(\cdot, t)-v^{\varepsilon_{N}}(\cdot, t)\right\|_{L^{1}(x)} \leq & \varepsilon_{N}\left\|\sum_{j, k=1}^{d} \partial_{j} R_{N}^{j, k}(\cdot, t) * \partial_{k} u_{N}(\cdot, t)\right\|_{L^{1}(x)} \\
& +\left\|\partial_{x} \cdot\left(I-P_{N}\right) f\left(u_{N}(\cdot, t)\right)\right\|_{L^{1}(x)}
\end{aligned}
$$


Using (3.3) with $r=s=1$, we have that the first term on the right does not exceed

$$
\begin{aligned}
& \varepsilon_{N}\left\|\sum_{j, k=1}^{d} \partial_{j} R_{N}^{j, k}(\cdot, t) * \partial_{k} u_{N}(\cdot, t)\right\|_{L^{1}(x)} \\
& \quad \leq \varepsilon_{N}\left\|\sum_{j, k=1}^{d} \partial_{j} R_{N}^{j, k}(\cdot, t)\right\|_{L^{1}(x)} \cdot\left\|\partial_{k} u_{N}(\cdot, t)\right\|_{L^{1}(x)} \\
& \quad \leq \text { Const } \varepsilon_{N} m_{N}(\log N)^{d} \cdot\left\|u_{N}(\cdot, t)\right\|_{B V(x)} \leq \text { Const } \sqrt{\varepsilon_{N}} .
\end{aligned}
$$

With this in mind, we can integrate (6.4) to obtain

$$
\begin{aligned}
& \left\|u_{N}(\cdot, t)-v^{\varepsilon_{N}}(\cdot, t)\right\|_{L^{1}(x)} \\
& \quad \leq \text { Const } \sqrt{\varepsilon_{N}}+\sqrt{2 \pi t}\left\|\partial_{x} \cdot\left(I-P_{N}\right) f\left(u_{N}\right)\right\|_{L^{2}(x,[0, t])} .
\end{aligned}
$$

According to $(2.8 \mathrm{~b})$, the second term on the right is the spectrally small truncation error

$$
\begin{array}{r}
\left\|\partial_{x} \cdot\left(I-P_{N}\right) f\left(u_{N}\right)\right\|_{L^{2}(x,[0, t])} \leq \text { Const } \mathscr{K}_{s} N^{-\left(s_{1}+\frac{\theta}{2}\right)}, \\
s_{1}=(1-\theta) s-1,
\end{array}
$$

which does not exceed $\mathscr{O}\left(\sqrt{\varepsilon_{N}}\right)$ for $s$ large enough $\left(s>\frac{1}{1-\theta}\right)$.

We summarize what we have shown in (6.2), (6.6), and (6.7), by stating the following theorem.

Theorem 6.1. Let $u_{N}$ be the $S V$ solution of $(1.2 \mathrm{a})-(1.2 \mathrm{~d})$, subject to the initial conditions (2.7). Then $u_{N}$ converges to the unique entropy solution of (1.1a)(1.1b), and the following error estimate holds:

$$
\left\|u_{N}(\cdot, t)-u(\cdot, t)\right\|_{L^{1}(x)} \leq \text { Const } \sqrt{\varepsilon_{N}}, \quad 0 \leq t \leq T
$$

\section{Proof of the MAIN A PRIORI estimates}

In this section we collect the promised proofs for the main a priori estimates associated with the SV approximation (1.2a), which we rewrite as

(7.1) $\partial_{t} u_{N}+\partial_{x} \cdot f\left(u_{N}\right)-\varepsilon_{N} \Delta u_{N}=\varepsilon_{N} \sum_{j, k=1}^{d} R_{N}^{j, k} * \partial_{j k}^{2} u_{N}+\partial_{x} \cdot\left(I-P_{N}\right) f\left(u_{N}\right)$

An upper bound on the first term on the right was provided in $\S 3$. We now turn to estimate the second term on the right of (7.1). Here, we first prepare the proof of (2.2), which we state as follows.

Theorem 7.1. The following estimate holds:

(7.2) $\left\|\partial_{x}^{s} \cdot f\left(u_{N}\right)\right\| \leq \mathscr{K}_{s}\left\|\partial_{x}^{s} u_{N}\right\|, \quad \mathscr{K}_{s} \sim \sum_{k=1}^{s}|f|_{C^{k}} \cdot\left|u_{N}\right|_{L^{\infty}}^{k-1}, \quad s=1,2, \ldots$ 
Proof. By the chain rule we have

$$
\begin{aligned}
\partial_{j}^{s} f(u)=\sum_{\{\alpha, \beta>0 \mid \alpha \cdot \beta=s\}} c_{\beta}\left(\partial_{j}^{\alpha_{1}} u\right)^{\beta_{1}}\left(\partial_{j}^{\alpha_{2}} u\right)^{\beta_{2}} \ldots\left(\partial_{j}^{\alpha_{d}} u\right)^{\beta_{d}}, & \\
& c_{\beta} \sim \partial_{u}^{r} f(u), \quad r=\sum_{k=1}^{d} \beta_{k} .
\end{aligned}
$$

The Hölder inequality followed by the Gagliardo-Nirenberg inequality implies that a typical term on the right does not exceed

$$
\begin{aligned}
& \left\|\left(\partial_{j}^{\alpha_{1}} u\right)^{\beta_{1}}\left(\partial_{j}^{\alpha_{2}} u\right)^{\beta_{2}} \ldots\left(\partial_{j}^{\alpha_{d}} u\right)^{\beta_{d}}\right\| \\
& \leq \prod_{k=1}^{d}\left\|\partial_{j}^{\alpha_{k}} u\right\|_{L^{\theta_{k} \beta_{k}}}^{\beta_{k}} \quad\left(\text { with } \theta_{k} \equiv \frac{s}{\alpha_{k} \beta_{k}}\right) \\
& \leq \text { Const } \prod_{k=1}^{d}\|u\|_{L^{\infty}}^{\left(1-\lambda_{k}\right) \beta_{k}} \cdot\left\|\partial_{j}^{s} u\right\|^{\lambda_{k} \beta_{k}} \quad\left(\text { with } \lambda_{k}=\frac{\alpha_{k}}{s}\right) \\
& \leq \text { Const }|u|_{L^{\infty}}^{r-1} \cdot\left\|\partial_{j}^{s} u\right\|, \quad r=\sum_{k=1}^{d} \beta_{k} \leq s,
\end{aligned}
$$

and hence (7.2) follows with $\mathscr{K}_{s} \sim \sum_{k=1}^{s}|f|_{C^{k}} \cdot|u|_{L^{\infty}}^{k-1}$.

Equipped with Lemma 7.1, we are now ready for the proof of the main result of $\S 2$, claiming that the smallest scale of the SV approximation is of order $\varepsilon_{N}$,

$$
\text { (7.3) } \varepsilon_{N}^{s}\left\|\partial_{x}^{s} u_{N}(\cdot, t)\right\|_{L^{2}(x)}+\varepsilon_{N}^{s+\frac{1}{2}}\left\|\partial_{x}^{s+1} u_{N}\right\|_{L^{2}(x,[0, t])} \leq \mathscr{B}_{s}+\varepsilon_{N}^{s}\left\|\partial_{x}^{s} u_{N}(\cdot, 0)\right\|_{L^{2}(x)} \text {. }
$$

Proof of Theorem 2.1. Spatial integration of (7.1) against $u_{N}(x, t)$ yields

$$
\begin{aligned}
\frac{1}{2} \frac{d}{d t}\left\|u_{N}\right\|^{2}+\varepsilon_{N}\left\|\partial_{x} u_{N}\right\|^{2} \leq & \left\|u_{N}\right\| \cdot \varepsilon_{N}\left\|\sum_{j, k=1}^{d} \partial_{j k}^{2} R_{N}^{j, k} * u_{N}\right\| \\
& +\sum_{j=1}^{d}\left\|\partial_{j} u_{N}\right\| \cdot\left\|\left(I-P_{N}\right) f^{j}\left(u_{N}\right)\right\| .
\end{aligned}
$$

Using (3.6) with $p=2$ for the first term on the right, and (2.3) with $(r, s)=$ $(0,1)$ for the second term, we find

$$
\begin{aligned}
\frac{1}{2} \frac{d}{d t}\left\|u_{N}\right\|^{2}+\left(\varepsilon_{N}-\frac{\mathscr{K}_{1}}{N}\right)\left\|\partial_{x} u_{N}\right\|^{2} \leq c_{N}\left\|u_{N}\right\|^{2}, & \\
& c_{N} \sim \varepsilon_{N} \cdot m_{N}^{2}(\log N)^{d} \leq \text { Const. }
\end{aligned}
$$

Hence, (7.3) follows for $s=0$ (with $\mathscr{K}_{0}=0$ ). 
The general case follows by induction on $s$. Spatial integration of (4.3a) against $\partial_{x}^{2 s} u_{N}(x, t)$ yields

$$
\begin{aligned}
\frac{1}{2} \frac{d}{d t}\left\|\partial_{x}^{s} u_{N}\right\|^{2}+\varepsilon_{N}\left\|\partial_{x}^{s+1} u_{N}\right\|^{2} \leq & \left\|\partial_{x}^{s} u_{N}\right\| \cdot \varepsilon_{N}\left\|\sum_{j, k=1}^{d} \partial_{j k}^{2} R_{N}^{j, k} * \partial_{x}^{s} u_{N}\right\| \\
& +\left\|\partial_{x}^{s+1} u_{N}\right\| \cdot\left\|\partial_{x}^{s-1} \partial_{x} \cdot P_{N} f\left(u_{N}\right)\right\| .
\end{aligned}
$$

Using (3.6) again and the Cauchy-Schwarz inequality to bound from above, respectively, the first and second term on the right of (7.4), we end up with

$$
\frac{d}{d t}\left\|\partial_{x}^{s} u_{N}\right\|^{2}+\frac{\varepsilon_{N}}{2}\left\|\partial_{x}^{s+1} u_{N}\right\|^{2} \leq \text { Const }\left\|\partial_{x}^{s} u_{N}\right\|^{2}+\frac{1}{2 \varepsilon_{N}}\left\|\partial_{x}^{s} \cdot P_{N} f\left(u_{N}\right)\right\|^{2}
$$

It remains to estimate the spatial derivatives of $P_{N} f\left(u_{N}\right)$. To this end, we use (2.2) and (2.3) to obtain

$$
\begin{aligned}
\left\|\partial_{x}^{s} \cdot P_{N} f\left(u_{N}\right)\right\| & \leq\left\|\partial_{x}^{s} \cdot f\left(u_{N}\right)\right\|+\left\|\partial_{x}^{s} \cdot\left(I-P_{N}\right) f\left(u_{N}\right)\right\| \\
& \leq \mathscr{K}_{s}\left\|\partial_{x}^{s} u_{N}\right\|+\frac{\mathscr{K}_{s+1}}{N}\left\|\partial_{x}^{s+1} u_{N}\right\| .
\end{aligned}
$$

Together with (7.5), we find

$$
\frac{d}{d t}\left\|\partial_{x}^{s} u_{N}\right\|^{2}+\left(\frac{\varepsilon_{N}}{2}-\frac{\mathscr{K}_{s+1}^{2}}{N^{2} \varepsilon_{N}}\right)\left\|\partial_{x}^{s+1} u_{N}\right\|^{2} \leq\left(\text { Const }+\frac{\mathscr{K}_{s}^{2}}{\varepsilon_{N}}\right)\left\|\partial_{x}^{s} u_{N}\right\|^{2} .
$$

In view of the SV parametrization in (2.4), temporal integration of the last inequality yields

$\left\|\partial_{x}^{s} u_{N}(\cdot, t)\right\|^{2}+\frac{1}{4} \varepsilon_{N}\left\|\partial_{x}^{s+1} u_{N}\right\|_{L^{2}(x,[0, t])}^{2} \leq \frac{\mathscr{K}_{s}^{2}}{\varepsilon_{N}}\left\|\partial_{x}^{s} u_{N}\right\|_{L^{2}(x,[0, t])}^{2}+\left\|\partial_{x}^{s} u_{N}(\cdot, 0)\right\|^{2}$,

and the result (7.3) follows by induction on $s$.

\section{ACKNOWLEDGMENTS}

Gui-Qiang Chen's research was supported in part by the Applied Mathematical Sciences subprogram of the Office of Energy Research, U.S. Department of Energy, under Contract W-31-109-Eng-38; by ONR Grant N00014-91-J-1384; and by an Alfred P. Sloan Foundation Fellowship. Eitan Tadmor's research was supported in part by ONR Grant N00014-91-J-1076.

\section{BIBLIOGRAPHY}

1. C. Canuto, M. Y. Hussaini, A. Quarteroni, and T. Zang, Spectral methods in fluid dynamics, Springer-Verlag, New York, 1988.

2. G.-Q. Chen, The compensated compactness method and the system of isentropic gas dynamics, Preprint MSRI-00527-91, Mathematical Science Research Institute, Berkeley, October 1990.

3. Rational Mech. Anal. 121 (1992), 131-185.

4. M. G. Crandall and A. Majda, Monotone difference approximations for scalar conservation laws, Math. Comp. 34 (1980), 1-21.

5. R. DiPerna, Measure-valued solutions to conservation laws, Arch. Rational Mech. Anal. 88 (1985), 223-270. 
6. Convergence of approximate solutions to conservation laws, Arch. Rational Mech. Anal. 82 (1983), 27-70.

7. D. Gottlieb and S. Orszag, Numerical analysis of spectral methods: Theory and applications, CBMS-NSF Regional Conference Series in Appl. Math., vol. 25, SIAM, Philadelphia, PA, 1977.

8. D. Gottlieb and E. Tadmor, Recovering pointwise values of discontinuous data within spectral accuracy, Progress in Supercomputing in Computational Fluid Dynamics, Progress in Scientific Computing, vol. 6 (E. M. Murman and S. S. Abarbanel, eds.), Birkhäuser, Boston, 1985, pp. 357-375.

9. H.-O. Kreiss, Fourier expansions of the solutions of Navier-Stokes equations and their exponential decay rate, Analyse Mathématique et Appl., Gauthier-Villars, Paris, 1988, pp. 245-262.

10. H.-O. Kreiss and J. Oliger, Comparison of accurate methods for the integration of hyperbolic equations, Tellus 24 (1972), 199-215.

11. N. N. Kuznetsov, On stable methods for solving nonlinear first order partial differential equations in the class of discontinuous solutions, Topics in Numerical Analysis III (Proc. Roy. Irish Acad. Conf.), Trinity College, Dublin, 1926, pp. 183-192.

12. P. D. Lax, Hyperbolic systems of conservation laws and the mathematical theory of shock waves, CBMS-NSF Regional Conference Series in Appl. Math., vol. 11, SIAM, Philadelphia, PA, 1973.

13. Y. Maday and E. Tadmor, Analysis of the spectral vanishing viscosity method for periodic conservation laws, SIAM J. Numer. Anal. 26 (1989), 854-870.

14. R. Sanders, On convergence of monotone finite difference schemes with variable spatial differencing, Math. Comp. 40 (1983), 91-106.

15. S. Schochet, The rate of convergence of spectral-viscosity methods for periodic scalar conservation laws, SIAM J. Numer. Anal. 27 (1990), 1142-1159.

16. D. Serre, La compacité par compensation pour les systèmes hyperboliques nonlinéaires de deux equations à une dimension d'espace, J. Math. Pures Appl. 65 (1986), 423-468.

17. J. Smoller, Shock waves and reaction-diffusion equations, Springer-Verlag, New York, 1983.

18. A. Szepessy, Measure-valued solutions of scalar conservation laws with boundary conditions, Arch. Rational Mech. Anal. 107 (1989), 181-193.

19. E. Tadmor, Convergence of spectral methods for nonlinear conservation laws, SIAM J. Numer. Anal. 26 (1989), 30-44.

20. __ Shock capturing by the spectral viscosity method, Comput. Methods Appl. Mech. Engrg. 78 (1990), 197-208.

21. Semi-discrete approximations to nonlinear systems of conservation laws; consistency and $L^{\infty}$-stability imply convergence, ICASE Report No. 88-41, 1988.

22. - Total-variation and error estimates for spectral viscosity approximations, Math. Comp. 60 (1993), 245-256.

Department of Mathematics, The University of Chicago, Chicago, Illinois 60637

Department of Mathematics, Michigan State University, East Lansing, Michigan 48824

School of Mathematical Sciences, Tel-Aviv University, Tel-Aviv 69978, IsRael 